\title{
La tecnología en la aeronáutica en pro de las energias renovables y de la Bio- Sustentabilidad
}

\section{Aeronautics technology in favor of renewable energiesand sustainable Bio}

\author{
SANTANA-VÁZQUEZ, Olivia†*, LÓPEZ-MELENDREZ, J Jesús, ROSALES-OLIVARES, Jorge \\ Enrique y REYNOSO-VILLANUEVA, Miriam
}

Universidad Aeronautica en Querétaro. (UNAQ) Dependencia: CGUT

ID $1^{\text {er }}$ Autor: Olivia, Santana-Vázquez, / ORC ID: 0000-0002-1795-5319, CVU CONACYT ID: 900748

ID $1^{\mathrm{er}}$ Coautor: J Jesús, López-Melendrez / ORC ID: 0000-0002-1710-9999, CVU CONACYT ID: 944688

ID $2^{\text {do }}$ Coautor: Jorge Enrique, Rosales-Olivares / ORC ID: 0000-0001-9815-3205, CVU CONACYT ID: 473268

ID $3^{\text {er }}$ Coautora: Miriam, Reynoso-Villanueva / ORC ID: 0000-0003-3307-9686, CVU CONACYT ID: 633822

DOI: $10.35429 /$ JSI.2019.10.3.20.28

Recibido 20 de Abril, 2019; Aceptado 30 de Junio, 2019

\section{Resumen}

Las ciencias básicas, la tecnología y las ciencias humanas en la aeronáutica se integran en favor de las energías renovables para del desarrollo sustentable. Es así que el objetivo de este proyecto es crear un sistema de almacenamiento de agua pluvial y condensada con riego automatizado, alimentado con energía solar que provea la humedad de cultivos y contribuya a la reducción de $\mathrm{CO} 2$ en la región de semidesierto de Querétaro. La metodología utilizada es investigación-acción, la cual consiste en observar y detectar una problemática real, enseguida analizar y valorar las posibilidades de intervención. en este caso fue es el desaprovechamiento de recursos naturales en la universidad (agua y energía solar). Enseguida se diseña una estrategia de acción la cual consistió en :1. Integrar un equipo de profesores especialistas y estudiantes .2. Planeación general del proyecto.3. Investigación teórica y de campo. 4. Diseño de sistemas: El hidráhulico, de estructuras, de riego automatizado con energía solar. 5 Aplicación de pruebas de dispositivos y el estudio de proceso de cultivos y de elaboración de composta. En este proyecto la contribución es sobretodo social y ecológica y no a la ciencia; se fomenta la responsabilidad social universitaria en la importancia de que la aplicación de la tecnología ofrezca alternativas para cuidado y uso de recursos naturales y un mayor uso de energías renovables. Como valor agregado se contribuye en la investigación aplicada con enfoque interdisciplinario de profesores y estudiantes de diferentes carreras aeronáuticas en pro del desarrollo sustentable. Se aclara que en esta etapa inicial aun no se hace aporte cietífico ya que se tiene planeado en una siguiente hacer pruebas y muestra de niveles de radiación y de humedad en la región.

Tecnología, Energia Renovable, Sustentailidad

\begin{abstract}
Basic sciences, technology and human sciences in aeronautics are integrated in favor of renewable energies for sustainable development.Thus, the objective of this project is to create a rainwater and condensed water storage system with automated irrigation, powered by solar energy that provides crop moisture and contributes to the reduction of $\mathrm{CO} 2$ in the semi-desert region of Querétaro. The methodology used is action research, which consists of observing and detecting a real problem to immediately analyze and assess the possibilities of intervention. In this case it was the waste of natural resources in the university (water and solar energy). An action strategy was designed afterwards which consisted in: 1 . Integration of a team of specialist teachers and students. 2. General planning of the project. 3. Theoretical and field research. 4. Systems design: hydraulic, structures, automated irrigation with solar energy. 5.Application of device tests and the study of the process of crop growing and composting. Our contribution in this project is above all social and ecological and not to science; university social responsibility is encouraged emphasizing the importance that the application of technology offer alternatives for the care and use of natural resources and greater use of renewable energy. As an added value, the project contributes to applied research with an interdisciplinary approach of professors and students of different aeronautical careers in favor of sustainable development. It is clarified that during this initial stage there is still no scientific contribution made as we plan, in a subsequent stage, to test and show levels of radiation and humidity in the region.
\end{abstract}

Technology, Renewable energy, Sustainable Bio

Citación: SANTANA-VÁZQUEZ, Olivia, LÓPEZ-MELENDREZ, J Jesús, ROSALES-OLIVARES, Jorge Enrique y REYNOSO-VILLANUEVA, Miriam. La tecnología en la aeronáutica en pro de las energias renovables y de la BioSustentabilidad. Revista de Innovación Sistemática. 2019. 3-10: 20-28

\footnotetext{
*Correspondencia al Autor (Correo electrónico: Olivia.santana@unaq.edu.mx)

$\uparrow$ Investigador contribuyendo como primer Autor.
} 


\section{Introducción}

La ciencia y la tecnología tienen como fin el desarrollo y bienestar de la humanidad, es así que este proyecto interdisciplinario hace una integración y aplicación de distintas ciencias y disciplinas tecnológicas. El objetivo es construir un biohuerto autosustentable en el cual se aprovecharán los recursos naturales (agua pluvial, agua condensada, energía solar, residuos orgánicos) y los estructurales (techos amplios y canaletas) de las naves de la Universidad Aeronáutica en Querétaro.

El valor agregado es que se constituye en un modelo ecológico de tecnología aplicada en el aprovechamiento de recursos en medio de la comunidad semirural y empresas aeronáuticas del entorno, en las cuales se ha comenzado a dar a conocer el proyecto como una muestra de la responsabilidad social universitaria en la que se integra la triple hélice:ACADEMIA-SOCIEDADEMPRESA, mendiante la aplicación de conocimientos tecnológicos para ofrecer alternativas al problema de escasez de agua y poco aprovechamiento de la energía solar los municipios del Marques y de Colón del semidesierto queretano.

La primera característíca es la integración de las áreas tecnológicas aeronauticas de Aviónica y Manufactura para desarrollar el sistema hidráulico de captación y almacenamiento de agua pluvial y condensada y el sistema de riego automatizado, ambos activados con energía solar; la segunda es el estudio estructural de los techos de las naves de la universidad donde la caída libre de agua fluye por canaletas que facilitan su almacenamiento; la tercera es la estética estructural del invernadero la cual se ha diseñado con motivo aeronáutico, una aeronave, (Aerohuerto).

También como característica es que además de las hortalizas que se cultivarán al el interior del invernadero en el exterior se ha reservado espacio para cultivo de cactáceas y otras especies como plátano, el cual servirá como materia prima para la investigación aeronáutica en el área de los materiales compuestos y la última característica valiosa, el gran impacto sociale al interior de la comunidad universitaria,en las familias,en las empresas y escuelas que a lo largo del año visitan la UNAQ ( Universidad Aeronáutica en Querétaro).
Por sus sistemas hidráhulico, eléctrico y electrónico se caracteriza como un modelo didáctico para concientizar en la aplicación de tecnología en pro del uso y cuidado del agua, el aprovechamiento de recursos, el fomento del uso de energías limpias, el cultivo de productos orgánicos, la salud nutricional y una economía sostenible.

Se parte de una problemática social observada en la zona del semi-desierto queretano de la cual la universidad y empresas por su ubicación forman parte, (crisis de agua y poco aprovechamiento de energía solar, agua pluvial y agua condensada).

Hipótesis: El aprovechamiento de la energía solar, el agua pluvial y condensada mediante la aplicación de tecnología interdisciplinaria en la construcción de biohuertos autosustentables coadyuvará a la responsabilidad social de la universidad, empresas y comunidades.

La primera sección del articulo hace referencia al estudio de los factores geofisicos (clima, temperatura, tipo de suelo, agotamiento de mantos acuiferos de la zona y las características físicas en la UNAQ, lugar donde se desarrolla el proyecto.

La segunda sección presenta el diseño de la estructura del prototipo del invernadero con la primera cisterna, el uso y aprovechamiento de la energía solar como una de las mejores opciones para activar el sistema de riego automatizado.

Y la tercera describe y muestra la fabricación de jardineras y el proceso de elaboración de composta para el cultivo de hortalizas.

\section{Los factores geofísicos en el semidesierto queretano de Colón Querétaro}

En Querétaro se comienza a padecer cada vez más la escasez de agua y como academia queremos colaborar en la concietización de su cuidado. Referente a este tema Morales (2017) en su artículo periodístico refiere "El agua es necesaria para todas las formas de vida... y es imprescindible generar conciencia entre la ciudadanía sobre su importancia, uso moderado, reuso y cuidado". La Organización Mundial de la Salud (OMS) estima que el uso inadecuado del agua derivará en que para 2025 la mitad de la población mundial (3 mil 500 millones de personas) viva en zonas con extrema escasez de agua.

SANTANA-VÁZQUEZ, Olivia, LÓPEZ-MELENDREZ, J Jesús, ROSALES-OLIVARES, Jorge Enrique y REYNOSO-VILLANUEVA, Miriam. La tecnología en la aeronáutica en pro de las energias renovables y de la Bio-Sustentabilidad. Revista de Innovación Sistemática. 2019 
En Querétaro, existe una sobreexplotación de acuíferos, al respecto el observatorio ciudadano dice: "seis de los 12 acuíferos existentes en el estado están sobreexplotados". El Observatorio Ciudadano de Protección Ambiental de Querétaro (OCPAQ). afirma. "En el acuífero de San Juan del Río se tiene una sobreexplotación de $133 \mathrm{~m}^{3}$, en el de Amazcala de $24.68 \mathrm{~m}^{3}$, para el ubicado en Buenavista la sobreexplotación es de 11.41 $m^{3}$, mientras que para los ubicados en Huimilpan, Tolimán y Querétaro, se calcula una sobreexplotación de $0.53 \mathrm{~m}^{3}, 0.70 \mathrm{~m}^{3}$ y 67 $m^{3}$ respectivamente, agrega Urribarren. "Esto genera un total de $237.32 \mathrm{~m}^{3}$ al año ". Con base a esta información nuestro proyecto se suma a quienes quieren ofrecer alternativas viables de solución a la problemática del agua en nuestra región.

\section{Respecto a las condiciones climatológicas en la zona de Cólón Querétaro}

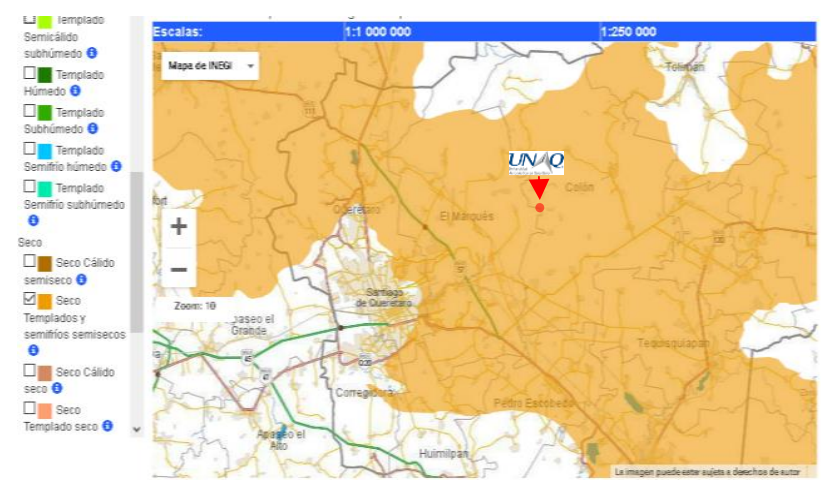

Figura 1 Mapa sobre los climas en el estado de Querétaro Fuente INEGI

Las condiciones climatológicas: " $\mathrm{El}$ municipio Colón, encontramos que éste se caracteriza por el clima de estepa local, INEGI, (2017). La ubicación geográfica de la Universidad Aeronáutica en Querétaro es de $20^{\circ} 37^{\prime} 32.5^{\prime \prime} \mathrm{N}$ y $100^{\circ} 11^{\prime} 15.3^{\prime} \mathrm{W}$ y a 1919 metros sobre el nivel del mar. Esta zona entre los municipios de Colón y el Marques se caracteriza por tener un clima de seco a semiseco, que a su vez representa el 51\% del estado de Querétaro.

Los grados de humedad son alrededor del 22.9\%, adicionalmente presenta una precipitación anual que va de los 550 a 600mm al año, ocasionando que sea una zona poco fértil para cualquier tipo de cultivo y con requerimientos altos de agua. Ver Fig. 2.

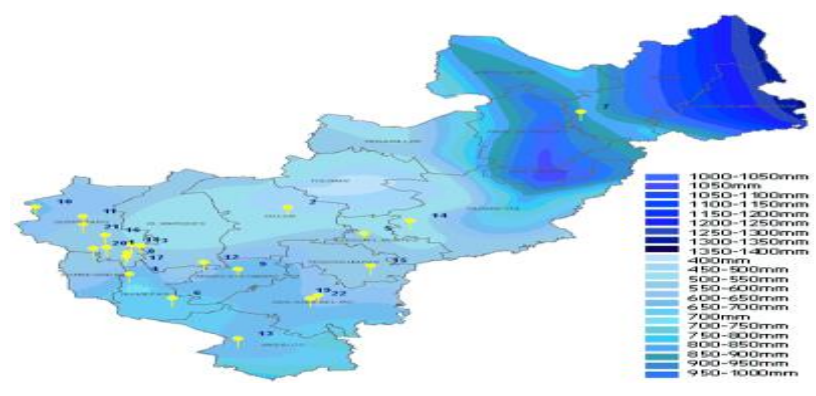

Figura 2 Taza de precipitación de agua pluvial en Querétaro-México

Fuente: INEGI

Durante el periodo que no llueve, la sostentabilidad del agua de riego queda a cargo de la condensación de la humedad que existe en el aire expuesto y al descenso de la temperatura que caracteriza a la zona. Es así que el agua condensada que corre sobre el techo de la nave de la UNAQ a través del sistema de canaletas de desagüe se aprovechará para el riego. Ver Fig.3.

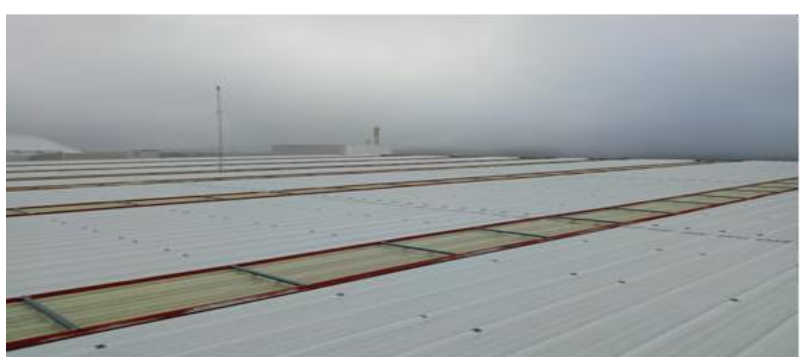

Figura 3 Superficie de techo de la UNAQ. Con canaletas hacia los desagues

La temperatura, en época de verano llega a registrar hasta $32^{\circ} \mathrm{C}$ y los índices de radiación UV son mayores a 10 , lo que equivale a una potencia solar de $1 \mathrm{~kW} / \mathrm{m} 2$; estos datos indican que la poca humedad que se deposita en el terreno se evapora rápidamente a causa de que el suelo es compacto y con poca absorción de líquidos, disminuyendo la humedad en el terreno; por ello que se considera una zona árida xerófila.

En la universidad cuando llueve es observable la cantidad de agua que corre sobre el grande techo de la nave principal, éste tiene una superficie perimetral de $703 \mathrm{~m}$, y un área de 1.92 héctareas. Obsérvese la Fig.4.donde se aprecia el área mencionada, el tipo de suelo y las canaletas por donde descienden las aguas. 


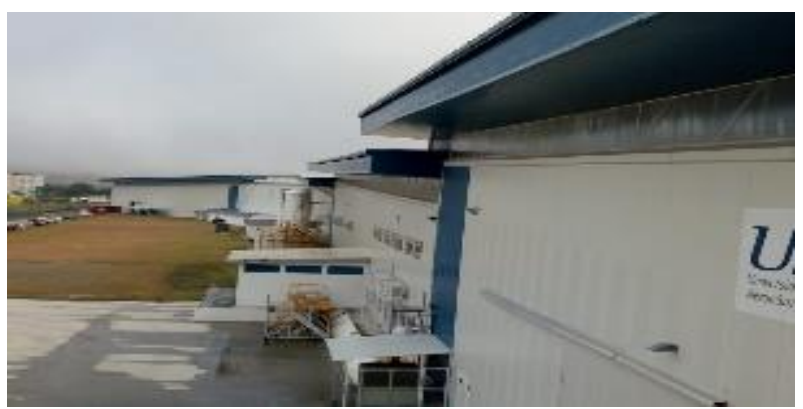

Figura 4 Area de la nave y caída de agua pluvial y condensada

Durante la noche y mañanas el agua condensada corre por las canaletas y sale por dos tuberías que la direccionan en caída libre sobre el campo.

\section{Resultados}

La universidad se encuentra ubicada geográficamente en el Municipio de Colón colindando con el del Marques y su taza de precipitación anual es de las más bajas en el estado, de 450- $500 \mathrm{~mm}$. Esto nos coloca entre las zonas más áridas de la localidad estatal y entre las de mayor escasez de agua. Cuantitativamente en temporada fría de las 4:00 a 9:00 A.M en los meses de octubre a febrero en el desague de una de las canaletas se obtiene 1L. de captación de agua condensada por cada media hora lo cual en 5 horas un solo desague nos ofrece 10 litros de agua condensada diariamente.

\section{Estructura del prototipo "aerohuerto" con la primera cisterna y sistemas hidraúlico y solar}

Con base a la ubicación física de los desagües, la universidad otorgó para este proyecto un área de $100 \mathrm{~m} 2$. para el invernadero, ésta se encuentra sobre una plancha de tepetate de $1.5 \mathrm{~m}$ de profundidad, lo cual nos indica que no es apta para el cultivo y será necesario acondicionar el suelo. Ver Fig. 5

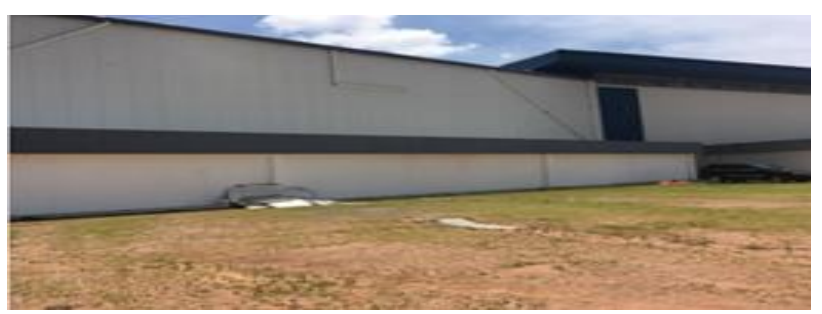

Figura 5 Area designada para el biohuerto UNAQ

Obsérvese el diseño del prototipo y la estructura del "domo", por su forma simula el fuselaje de una aeronave. Ver Fig 6.

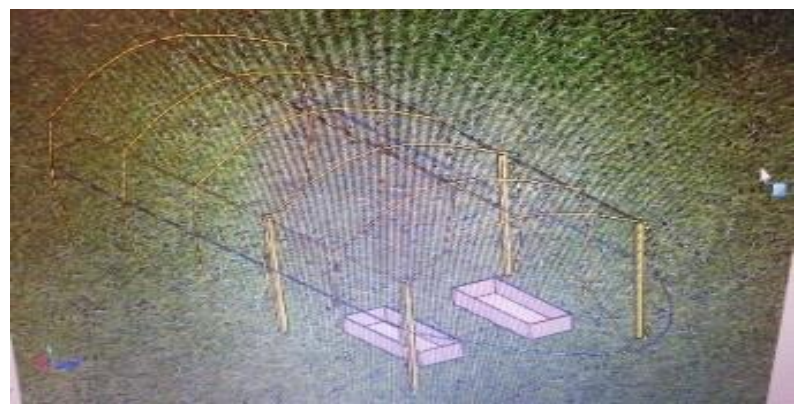

Figura 6 Prototipo del domo del aerohuerto UNAQ

Esta estructura está hecha de tubos de policloruro de vinilo (PVC); es un material ligero, resistente, inerte y completamente inocuo, con buen comportamiento al fuego y resistente a la intemperie. Se cubrirá con plástico lechoso de $30 \%$ de sombra el cual permite la entrada de luz de todas las longitudes de onda, para que las plantas puedan realizar la fotosíntesis. En algunos puntos del domo se colocaron columnas metálicas para soportar las cargas y esfuerzos que se lleguen a presentar. Ver Figura. 7.

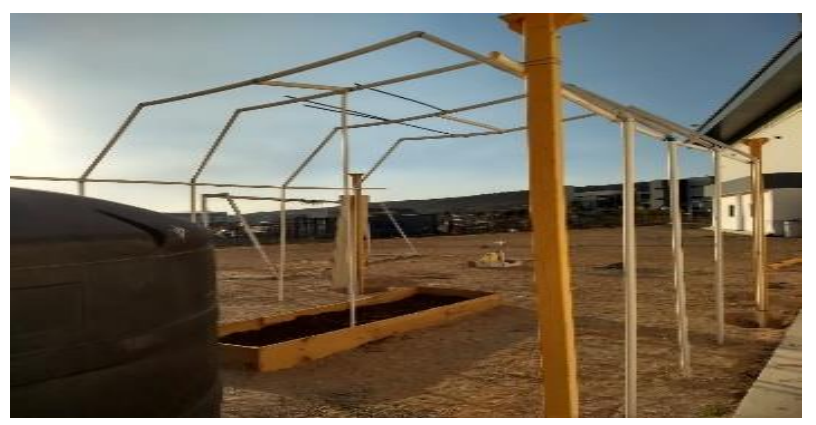

Figura 7 Estructura en PVC del domo con columnas metálicas de soporte

Sistema hidráulico: Se realizó el cálculo de tubería necesario para direccionar el agua al reservorio y lo requerido fueron 60 metros de tubería de PVC de 2 pulgadas de diámetro; este cálculo conlleva todos los accesorios que usarán en dicha instalación; también se calculó el peso de la cisterna con agua y accesorios en $5500 \mathrm{~kg}$, dato con el cual realizó la obra civil de una plancha de $3 \mathrm{~m} \times 3 \mathrm{~m}$, teniendo un área de $9 \mathrm{~m} 2$. de concreto armado para soportar la cisterna y que ésta no se fracture. Ver Figura. 8 


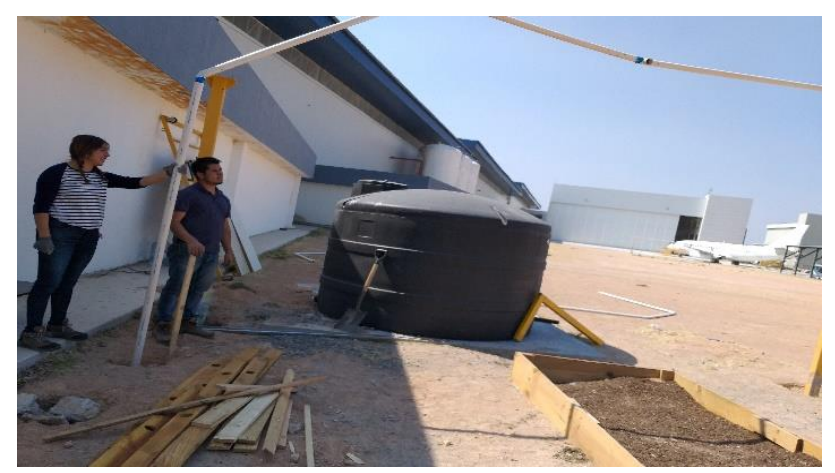

Figura 8 Primera cisterna de agua sobre la plancha construida

Finalmente, observése la Figura. 9. Que muestra la distribución general de las secciones de cultivo en jardineras sobre la cuales se distribuirá el riego automatizado desde la cisterna.

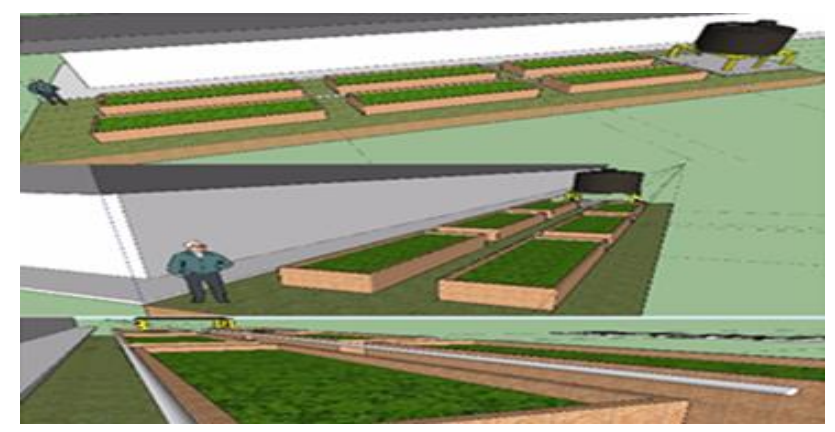

Figura 9 Distribución de áreas de cultivos para orientación de los sistemas hidrahulico y de riego

La SEMARNAT (2018), con respecto al uso de energías renovables afirma: "Representan mayor seguridad energética, menos contaminación y ahorros a la economía de estados y municipios". México destaca como territorio con mayor promedio de radiación solar anual con índices que van de los $4.4 \mathrm{kWh} / \mathbf{m} 2$ por día en la zona centro, a los $6.3 \mathrm{kWh} / \mathrm{m} 2$ por día en el norte del país. Ver figura 10.

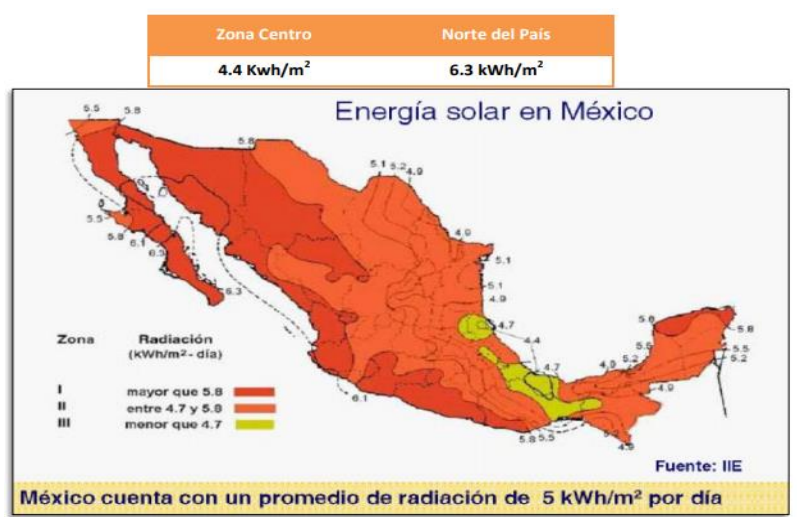

Figura 10 Radiación solar en la región del México Fuente: Instituto de investigaciones eléctricas
A lo largo de los meses del año el estado de Querétaro se coloca entre los de alta radiación solar llegando al nivel de 6.9 en el mes de mayo. Obsérvese la tabla 1.

\begin{tabular}{|l|l|l|l|l|l|l|l|l|l|l|l|l|}
\hline Ene & Feb & Mar & Abril & Maro & Lunio & Wio & Agosto & Sept. & Oct. & Nov. & Dic. & PROMEDlO \\
\hline 5.0 & 5.7 & 6.4 & 6.8 & 6.9 & 6.4 & 6.4 & 6.4 & 6.3 & 5.4 & 5.0 & 4.4 & 5.9 \\
& & & & & & & & & & & & \\
\hline
\end{tabular}

Tabla 1 Niveles de radiación solar en Querétaro Fuente: Instituto de investigación eléctrica

Es importante el aprovechamiento sustentable de la energía solar, es por eso que en este proyecto se diseñó el prototipo de riego automático con el uso de la energía solar. Este sistema se basa en la utilización de dos (2) módulos solares de 130 Vatios. Ver figura 11. Un (1) regulador de carga, un (1) inversor Xpower Digital 800w, dos (2) baterías de alta profundidad las cuales tienen una capacidad de $470.02 \mathrm{kWh}$, una (1) bomba sumergible de $0.5 \mathrm{HP}$ de potencia, una red de tuberías y 2 aspersores, los cuales se garantizarán el óptimo funcionamiento del sistema de riego que ofrece ahorro de agua y de energía eléctrica, beneficiando al medio ambiente y a la comunidad universitaria de la UNAQ.

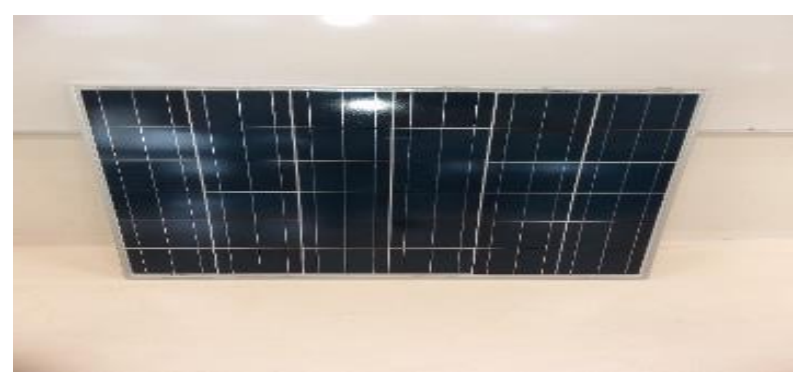

Figura 11 Panel solar que suministrará la energía para el sistema de riego automático

Riego Automatizado Autosustentable: De acuerdo al diseño que implica riego por goteo, este se controlará con una electroválvula que será energizada por una batería de 12VCD. Esta batería será cargada con una fuente de alimentación de aproximadamente de 22 VCD formada por un panel de celdas solares.

El sensado de la humedad en la tierra se realizará con una matriz de sensores los cuales serán alimentados también por la batería y enviarán la señal de requerimiento de humedad a la electroválvula. El agua que controla esta válvula será proporcionada por la fuerza de gravedad del líquido contenido en la cisterna de almacenamiento. 
El riego será por goteo y al tener la tierra la suficiente humedad se cerrará la válvula, permitiendo tener un buen control en la dosificación del agua. El sistema se autosustenta en el control y alimentación eléctrica, requiriendo poca atención humana. Ver tabla 2. de cálculo de voltajes requeridos.

\begin{tabular}{|c|c|}
\hline Datos & Medidas \\
\hline Voltaje máximo del panel solar & $22.4 \mathrm{Vmax}$. \\
\hline Voltaje máximo con la bomba & 20.2 Vmax. \\
\hline Voltaje máximo primer circuito & 19.9 Vmax. \\
\hline Voltaje máximo con relevador & $21.5 \mathrm{Vmax}$. \\
\hline
\end{tabular}

Tabla 2 Voltajes requeridos para el sistema de riego automatizado

A continuación, se describe el funcionamiento de circuitos con los sensores de humedad requeridos en el sistema de riego automatizado.

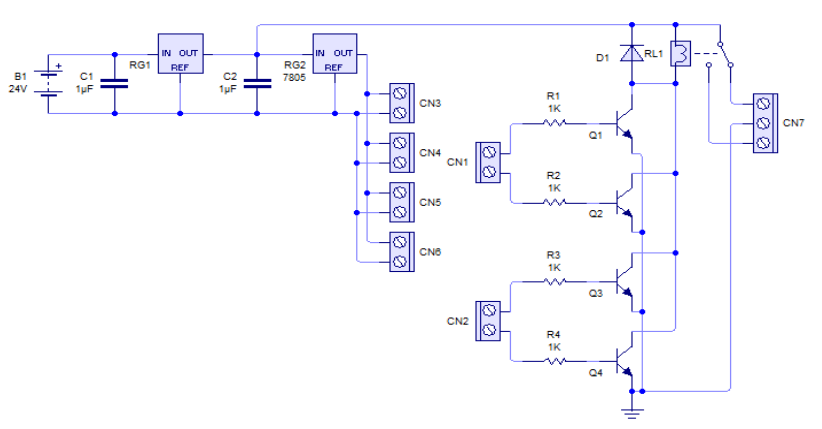

Figura 12 Circuito para la detección de humedad

\section{Funcionamiento del circuito}

El panel solar suministrará en condiciones adecuadas entre 22 a 18 voltios de corriente directa, provocando que la batería esté siempre cargándose, cuando el panel solar deje de suministrar energía, la batería que todo el día estuvo cargándose sustituirá al panel solar para que el circuito siempre esté funcionando sin la necesidad de una corriente exterior.

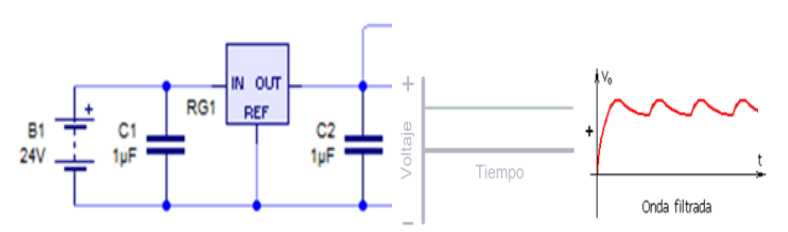

Figura 13 Regulación de voltaje y señal constante y Señal con rizado
El $\operatorname{lm} 7812$ su función es que el voltaje suministrado por el panel solar o batería, lo regula a 12 voltios, los condensadores cerámicos se encargarán de que el voltaje de salida del regulador siempre se mande una señal constante. Ver. Figura. 13. Todo esto servirá para la etapa de alimentación del relevador y de la bomba de agua. Nota, para las primeras pruebas se cambio la bomba de agua por un led.

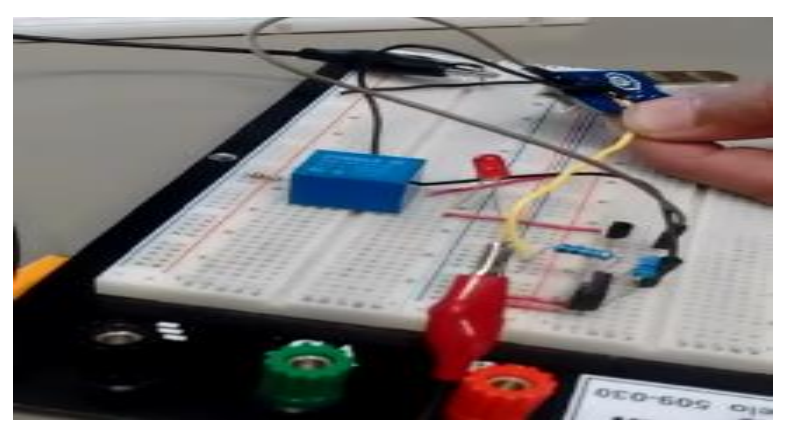

Figura 14 El regulador Im 7805 suministra energia a los sensores de humedad

El regulador $\operatorname{lm} 7805$ su función en este circuito es la suministración de 5 voltios a todos los sensores de humedad. Los terminales a bloques en el positivo y negativo para nuestros sensores. Ver Figura.15

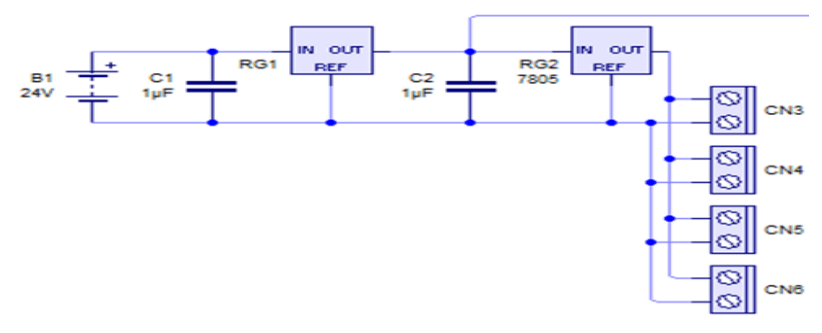

Figura 15 Bloques positivo y negativo

\section{Sensor de humedad}

\begin{tabular}{|l|l|l|}
\hline \multicolumn{2}{|c|}{ Datos } & \multicolumn{3}{|c|}{ Medidas } \\
\hline $\begin{array}{l}\text { Voltaje } \\
\text { alimentación }\end{array}$ & $\begin{array}{l}5 \text { volts. Etapa de control del } \\
\text { relevador para poder controlar la } \\
\text { bomba de agua }\end{array}$ \\
\hline $\begin{array}{l}\text { Voltaje de salida } \\
\text { máxima }\end{array}$ & 4.5 volts. \\
\hline $\begin{array}{l}\text { Voltaje de salida } \\
\text { mínima }\end{array}$ & 3.2 volts. \\
\hline
\end{tabular}

Tabla 3 Cálculo de Volts para sensores de humedad

El sensor de humedad que funciona con 5 voltios entrada estará en modo digital esto significará que nada más nos proporcionará un 1 cuando hay humedad y un cero cuando no hay humedad. Ver Figura. 16 


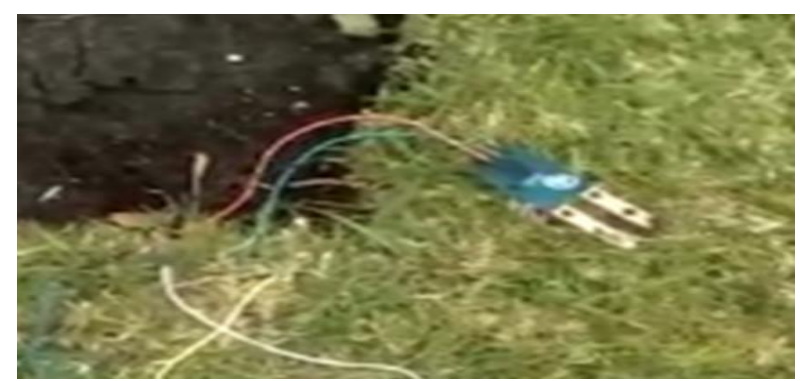

Figura 16 Prueba de humedad de suelo con sensores

El sensor de humedad nos dará aproximadamente entre 3.2 y 4.5 voltios cuando esté en uno y 0 voltios cuando está en cero.

Se utilizarán cuatro sensores, puestos en diferentes lugares para la detección de humedad $o$ agua en las jardineras.

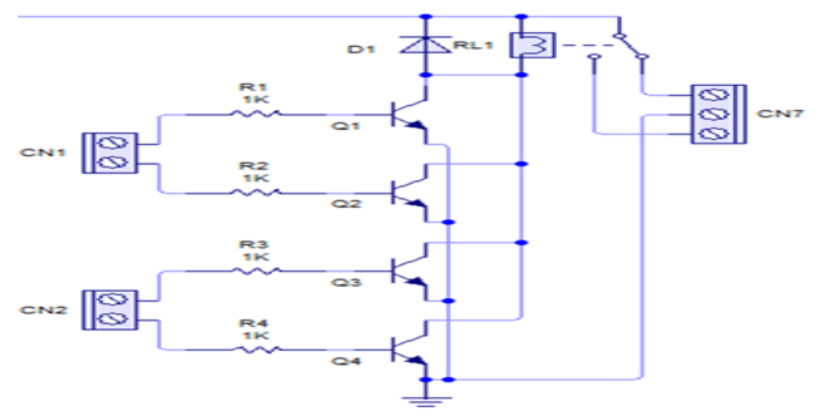

Figura 17 Etapa de control del relevador para poder controlar la bomba de agua

Una vez que cualquier sensor de humedad haya mandado un 1 , el transistor 2n2222a se polarizara su base dejando pasar el voltaje del colector al emisor, provocando que el relevador se active y con ello la bomba de agua. Ver Fg.17.

\section{Resultados}

1.Los niveles de radiación solar en Querétaro durante los meses del año aseguran una constante energización de los paneles solares.

2.Los sensores de humedad para su funcionamiento no rebasan los volts que almacena y suministra el panel solar por tanto para el riego un solo panel de 22 volts logra activar los sensores de humedad y la bomba.

\section{La fabricación de jardineras, cultivos y proceso de elaboración de composta}

En la $1^{a}$ fase de este proyecto se acondicionan las jardineras sobre el suelo y se manufacturan dos prototipos más: una de fibra de vidrio y otras del scraph de placas de aluminio. Ver Figura. 18.
También se definen los tipos de hortalizas que se cultivarán al interior del biohuerto: zanahoria, espinaca, rábano, lechuga, perejil, tomate, cebolla, menta, piña y albahaca. $\mathrm{Y}$ al exterior, plantio de cáctaseas las cuales se utilizarán para experimentos de investigación sobre materiales compuestos para el desarrollo aeronáutico.

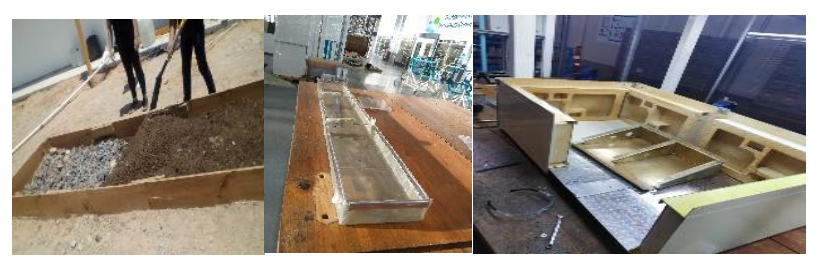

Figura18 Tipos de jardineras para el aeorohuerto

A la par de la fabricación de las jardineras se procesó la primera composta, la cual es el material orgánico (fertilizante natural) que se obtiene como producto de la acción microbiana controlada sobre residuos orgánicos. A) recaudación de residuos orgánicos de las cafeterías de la Universidad. B) En una caja de plástico se colocó en la parte inferior una bolsa negra para residuos y sobre ella se agregó una capa de tierra y agua para mantenerla húmeda. C) Adicionalmente, se agregó una capa de trozos de cartón encima de la tierra y una capa de residuos orgánicos. D) Inmediatamente, se comenzó a agregar una capa de material secó por dos capas de desechos orgánicos. E) Después, se agregó una capa de tierra y 1 litro de agua para humedecer. F) Finalmente, se tapó el contenedor y se dejó reposar por tres días, a partir de entonces el cuidado que se tuvo fue el siguiente: se agregó agua tres veces por semana y se mezcló constantemente durante un mes. (Ver Figura. 19 del proceso de composta.

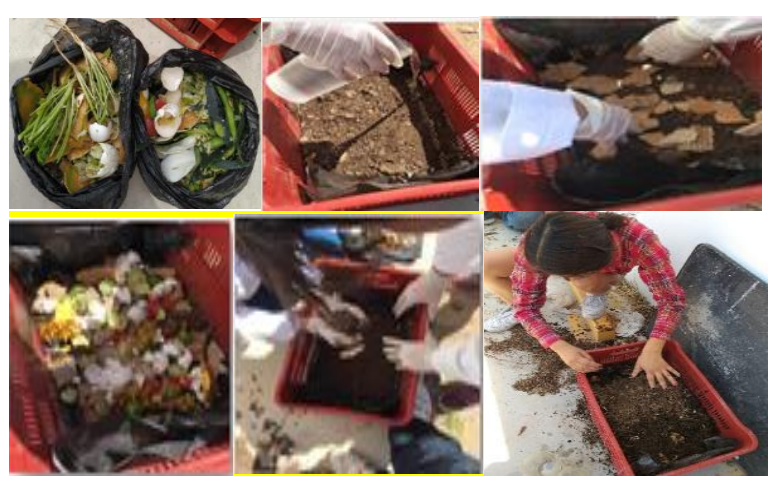

Figura 19 Proceso de elaboración de composta

SANTANA-VÁZQUEZ, Olivia, LÓPEZ-MELENDREZ, J Jesús, ROSALES-OLIVARES, Jorge Enrique y REYNOSO-VILLANUEVA, Miriam. La tecnología en la aeronáutica en pro de las energias renovables y de la Bio-Sustentabilidad. Revista de Innovación Sistemática. 2019. 


\section{Resultados}

El área del huerto al tener $1 \mathrm{~m}$ de profundidad en terreno industrializado a base de una capa de tepetate los cual nos indicó no era apto para cultivos, se está acondicionando el suelo con tierra de cultivos y humus orgánico. Cada jardinera requiere 7 costales de tierra para cultivo con $20 \mathrm{Kl} \mathrm{C} / \mathrm{U}$, lo que equivale $140 \mathrm{Kl}$.

A partir del scrap generado por las practicas realizadas en la universidad, se reutilizan varias placas y así poder realizar jardineras con elementos $100 \%$ reciclados, esto para la reducción de costos.

\section{La metodología}

Este proyecto en su primera etapa utiliza la metodología de investigación-acción que es más cualitativa que cuantitativa. Para ello se parte de datos generales de medición de escasez de agua en la región y la observación del desaprovechamiento permanente del agua condensada y pluvial en la UNAQ. Posteriormente se realiza el estado del arte geofísico del lugar, así como el diseño estructural del prototipo del biohuerto y su sistemas: La estructura del domo integra la construcción de un sistema hidráulico para almacenamiento del agua pluvial y condensada, seguido de esto se realiza el estudio y desarrollo de un sistema de riego automatizado con uso de energía solar y finalmente estudios del método para el cultivo y cuidado de hortalizas y el proceso de elaboración de composta con el diseño y la fabricación de composteros.

La técnica de captación de aguas se realiza mediante la utilización de las canaletas que están sobre la nave, para dar cause a las tomas que la conducirán a la cisterna de alamacenamiento; de la cual el sistema de riego automatizado se alimentará. Este último requiere de energía solar obtenida mediante los paneles con la finalidad de activar los sensores de humedad y los diferentes dispositivos, los cuales se están implementando. Recursos: Humanos: profesores especializados y estudiantes de manufactura y de aviónica. Materiales: de construcción (cemento, grava, arena, varilla. hidrahulicos: tubos, bombas, pegamentos, cisterna y accesorios...) Electricos: panel, sensores, placas, cables...) Naturales: residuos orgánicos. Estructuras: perfil, madera, recipientes-tambos. scrap de placas de aluminio, fibra de vidrio, resina, remaches, entre otros.
Desde su planteamiento contempla la difusión en las empresas del sector para que se sumen al cuidado y aprovechamiento del agua, así como a la aplicación de las energías renovables, en este caso la energía solar.

Sus impactos son: ecológico, social y económico ya que se utiliza la tecnología en favor del uso y cuidado de recursos naturales y esto favorece el desarrollo sustentable. También se impactará en la comunidad educativa mediante la concientización de mejora en la alimentación sana en consumo de verduras. Y un tercer impacto es en nuestro entorno comunitario e industrial, al presentar y promover el prototipo en las empresas cercanas y hacerles ver los beneficios ecológicos, económicos y sociales del cuidado y uso de recursos naturales.

Nota. Este proyecto, aunque no está concluido en todas sus fases y solamente en la primera ha sido presentado en la empresa Safrán, una de las más importantes del clúster aeronáutico en México y ha sido muy valorado por su interdisciplinariedad y su enfoque tecnológico, ecológico y social el cual desde la academia muestra un trabajo interdisciplinario entre estudiantes $y$ profesores asesores especializados en la industria y en la docencia. La Figura. 20. muestra la planeación general.

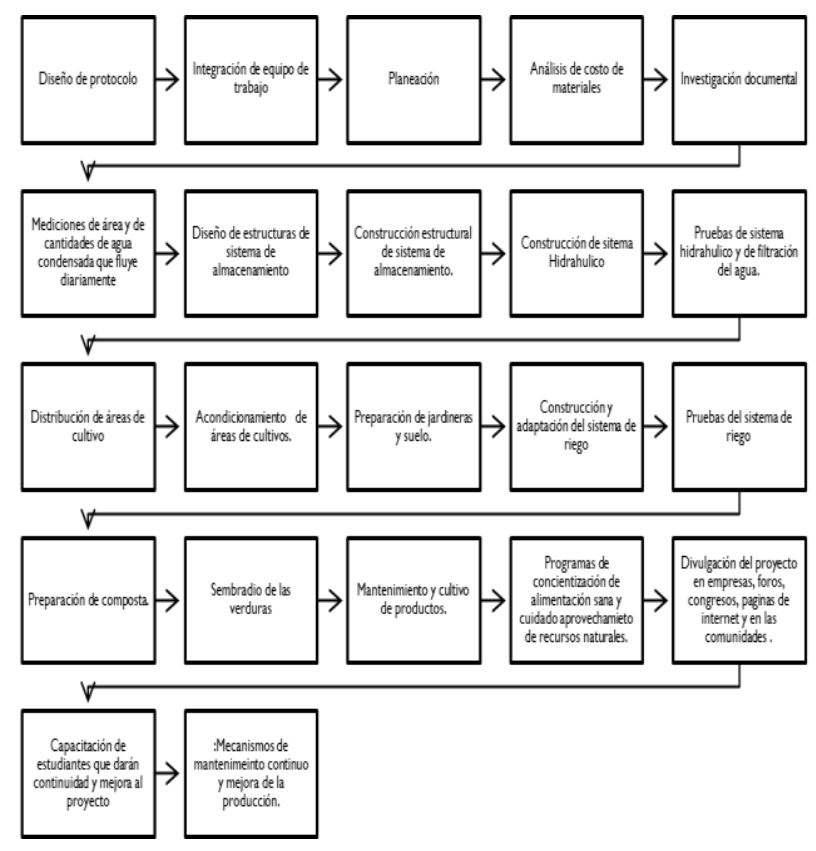

Figura 20 Planeación general metodológica de cada uno de los pasos del desarrollo de este proyecto 


\section{Conclusión}

Se puede concluir en esta primera etapa que el proyecto interdisciplinario fomenta la aplicación de la Ciencia y Tecnología en favor de la sustentabilidad ofreciendo alternativas a la problemática de escasez de agua y aprovechamiento de recursos naturales. Denotando la participación de las empresas en interacción con la academia mediante aplicación de tecnología en pro de la sustentabilidad.

\section{Los resultados obtenidos}

Los resultados obtenidos son variados y en cada sección se han explicado, pero en sintésis se puede decir que se ha provocado una perpectiva diferente en profesores y estudiantes en cuanto a la aplicación de los conocimientos tecnológicos. Las autoridades académicas y administrativas de la universidad dan muestra de un mayor interés por la responsabilidad social del aprovechamiento del agua. También se puede remarcar que la investigación sobre los factores geofísicos de la región ha dado mayor fundamento y soporte a la hipótesis planteada ante el problema identificado. Y en cuanto a la divulgación ésta se ha venido realizando a nivel estatal por CONCYTEQ y fue elegido a nivel Nacional para presentarse en el Concurso Nacional de Innovación y Emprendimiento ( CONIES) 2019.

\section{Posibilidad de mejora}

Darle un alcance más amplio mediante la instalación de otras cisternas con mayor capacidad de almacenamiento de agua pluvial para utilizar el agua en otros servicios de la universidad. Yse presentará en otras empresas aeronáuticas del entorno con fines de posicionamiento en el mercado.

\section{Agradecimiento}

A los estudiantes y profesores de la UNAQ que colaboran. Al CONCYTEQ quien a través del concurso de Nuevos Talentos eligió nuestro proyecto y otorgó cuatro becas a los estudiantes y un pequeño ingreso económico para efectuar la primera fase. También se agradece a los directivos y administrativos de la UNAQ quienes han dado la autorización para el desarrollo de este proyecto.

\section{Referencias}

INEGI (2014) Contenidos geográficos. En http://internet.contenidos.inegi.org.mx/contenid os/productos/prod_serv/contenidos/espanol/bvi negi/productos/historicos/2104/702825937539/ 702825937539_2.pdf. Recuperado 10/11/18.

INEGI (2017) Mapas de climatología de Querétaro. En http://www.beta.inegi.org.mx/temas/mapas/cli matologia/, consulta octubre 2018.

INEGI. (2017) Anuario estadístico y geográfico de Querétaro $2017 . \quad$ En https://www.datatur.sectur.gob.mx/ITxEF_Doc s/QRO_ANUARIO_PDF.pdf.

Instituto de Investigaciones eléctricas en https://www.gob.mx/ineel\#multimedia.

Recuperado 3/10/18.

Morales (2018) Escasez de agua, una problemática mundial. En https://amqueretaro.com/especiales/2018/07/09/ escasez-del-agua-una-problematica-mundial. Recuperado el 3/ 11/2018.

SEDESU (2017) E Servicios Ambientales Integrales de Consultoría y Cambio Climático. En SEDESU (2016) en http://www.queretaro.gob.mx/sedesu/contenido aspx?q=q05znG4LZgnZ8+zz89iPFSMkhLnC HX1. Recuperado 25/08/18.

SEMARNAT ( 2018) Energías renovables, gran oportunidad para México. EN https://www.gob.mx/semarnat/articulos/energia s-renovables-gran-oportunidad-para-mexico172759? idiom=es . Recuperado 5/11/18

SEMARNAT y CONAGUA. Normas oficiales mexicanas. NOM-001-SEMARNAT-1996. NOM-002-SEMARNAT-1996. NOM-003SEMARNAT-1997.

UNAM. El Potencial de la radiación solar. En http://www.ptolomeo.unam.mx:8080/xmlui/bits tream/handle/132.248.52.100/277/A5.pdf. $23 / 09 / 2018$ 